\title{
Extension of the millimeter- and submillimeter-wave spectral databases of deuterated methyl cyanides $\left(\mathrm{CH}_{2} \mathrm{DCN} \text { and } \mathrm{CHD}_{2} \mathrm{CN}\right)^{\star}$
}

\author{
L. Nguyen ${ }^{1,2}$, A. Walters ${ }^{1,2}$, L. Margulès ${ }^{3}$, R. A. Motiyenko ${ }^{3}$, J.-C. Guilleminn ${ }^{4}$, C. Kahane ${ }^{5}$, and C. Ceccarelli ${ }^{5}$ \\ 1 Université de Toulouse, UPS-OMP, IRAP, Toulouse, France \\ e-mail: linh.nguyen80@yahoo.fr; adam.walters@irap.omp.eu \\ 2 CNRS, IRAP, 9 avenue du Colonel Roche, BP 44346, 31028 Toulouse Cedex 4, France \\ ${ }^{3}$ Laboratoire de Physique des Lasers, Atomes, et Molécules, UMR CNRS 8523, Université de Lille 1, \\ 59655 Villeneuve d'Ascq Cedex, France \\ 4 Sciences Chimiques de Rennes, École Nationale Supérieure de Chimie de Rennes, CNRS, UMR 6226, Avenue du Général Leclerc, \\ CS 50837, 35708 Rennes Cedex 7, France \\ 5 UJF-Grenoble 1/CNRS-INSU, Institut de Planétologie et d'Astrophysique de Grenoble (IPAG), UMR 5274, \\ 38041 Grenoble Cedex 9, France
}

Received 24 July 2012 / Accepted 28 January 2013

\section{ABSTRACT}

\begin{abstract}
Context. The study of deuterated abundant molecules is recognized as important in understanding molecular complexity in starforming regions.

Aims. We seek to assign the laboratory millimeter and submillimeter wave rotational spectra of the $\mathrm{CHD}_{2} \mathrm{CN}_{\text {and }} \mathrm{CH}_{2} \mathrm{DCN}$ deuterated isotopologues of methyl cyanide over a wide frequency range in order to provide precise spectral predictions for observations. Methods. Using the solid-state submillimeter-wave spectrometer in Lille, we measured and assigned 723 new lines for $\mathrm{CHD}_{2} \mathrm{CN}$ and 307 new lines for $\mathrm{CH}_{2} \mathrm{DCN}$. The observed rotational transition frequencies were fitted with the ASFIT program to determine the spectroscopic parameters. The prediction of transition frequencies was performed using the SPCAT program.

Results. Measurements for both isotoplogues were taken up to $945 \mathrm{GHz}$ and are made available at the CDS. For $\mathrm{CHD}_{2} \mathrm{CN}$ this is significantly higher than the previous range of measurement up to $40 \mathrm{GHz}$. For $\mathrm{CH}_{2} \mathrm{DCN}$ many more lines of high $K_{a}$ have been measured than previously.

Conclusions. Our work confirms the recent analysis for $\mathrm{CHD}_{2} \mathrm{CN}$ given in the CDMS database and extends the number of determined molecular parameters from 10 to 19 . For $\mathrm{CH}_{2} \mathrm{DCN}, 3$ new parameters including $D_{K}$ have been determined, and the uncertainty on parameters has been decreased by a factor of approximately 2 . For both isotopologues the measured data show shifts in the frequency of some high $K_{a}$ transitions that are attributed to interactions with a low-lying vibrational state. The availability of more directly measured data and the increase in confidence of the predictions to higher quantum numbers and frequencies will be helpful for the radio astronomical detection of deuterated isotopologues of methyl cyanide in the interstellar medium.
\end{abstract}

Key words. ISM: molecules - methods: laboratory - line: identification - submillimeter: ISM - molecular data

\section{Introduction}

With its high abundance and symmetric top spectral pattern, methyl cyanide (or acetonitrile), $\mathrm{CH}_{3} \mathrm{CN}$, is known as an excellent probe-molecule for deriving the kinetic temperature and density of interstellar objects. It is thus not surprising that its rotational spectrum has been comprehensively studied (see Cazzoli \& Puzzarini 2006, for references and a very recently published paper of Müller et al. 2009). However, the ambition of astronomers does not stop at investigating abundant molecules like $\mathrm{CH}_{3} \mathrm{CN}$. The astrophysical study of the isotopologues of abundant molecules provides valuable clues for understanding the complex physical and chemical phenomena occurring in the interstellar medium (ISM). The average atomic deuterium-tohydrogen ratio in the local interstellar medium is around $10^{-5}$. However, exceptionally high deuterium-to-hydrogen ratios have been observed for certain molecules in hot cores and especially in hot corinos (Ceccarelli et al. 2007). This enrichment in deuterium is explained by reactions taking place both in the

* Full Tables B.1 and B.2 are only available in electronic form at the CDS via anonymous ftp to cdsarc.u-strasbg. $f r(130.79 .128 .5)$ or via

http://cdsarc.u-strasbg.fr/viz-bin/qcat?]/A+A/553/A84 gas phase and on the surface of grains, however, the enrichment appears to change from species to species and from one object to another, and the precise mechanisms taking place are still subject to debate. Observations of deuterated species are thus extremely valuable for constraining chemical models that may allow us to reconstruct the past history of protostars (Taquet et al. 2012). For this purpose, it is essential to know in advance precise rotational transition frequencies for these isotopologues, and other spectral information needed to model intensities. The necessary data is provided from laboratory measurements and their analysis, as reported here for $\mathrm{CH}_{2} \mathrm{DCN}$ and $\mathrm{CHD}_{2} \mathrm{CN}$.

Concerning the substituted ${ }^{13} \mathrm{C}$ and ${ }^{15} \mathrm{~N}$ isotopologues, after some investigations dating from the 1980's (Demaison et al. 1979; Boucher et al. 1980, etc.), Pearson \& Müller (1996) reported in 1996 the spectra of ${ }^{13} \mathrm{CH}_{3} \mathrm{CN}, \mathrm{CH}_{3}{ }^{13} \mathrm{CN}$, and $\mathrm{CH}_{3} \mathrm{C}^{15} \mathrm{~N}$ in the $294-607 \mathrm{GHz}$ frequency range. Recently, Müller et al. (2009) have extended the studied frequency range up to about $1.2 \mathrm{THz}$. In addition to the measurements of the singly substituted ${ }^{13} \mathrm{C}$ isotopologues, the previous publication also reports the high-resolution spectrum of the doubly substituted one (i.e. ${ }^{13} \mathrm{CH}_{3}{ }^{13} \mathrm{CN}$ ), for which only measurements up to $72 \mathrm{GHz}$ were previously available (Tam et al. 1988). 
As for laboratory measurements of deuterated methyl cyanide, the ground state microwave spectrum of $\mathrm{CD}_{3} \mathrm{CN}$ (triply deuterated methyl cyanide) was measured between 8 and $240 \mathrm{GHz}$ by Demaison et al. (1979). Later, Brown et al. (1989) extended the measurements to the submillimeter region. Moreover, the rotational spectrum of $\mathrm{CD}_{3} \mathrm{CN}$ in excited vibrational states has already been the subject of several publications (Matsumura et al. 1962; Cosleou et al. 1989, 1991; Chen et al. 1991).

In the present work, we focus on the millimeter and submillimeter spectra up to $945 \mathrm{GHz}$ of singly and doubly deuterated methyl cyanides, i.e. $\mathrm{CH}_{2} \mathrm{DCN}$ and $\mathrm{CHD}_{2} \mathrm{CN}$, in their ground vibrational states. Previous studies have been reported for these deuterated species but were limited to low-frequency measurements for $\mathrm{CHD}_{2} \mathrm{CN}$ and could be beneficially completed for $\mathrm{CH}_{2} \mathrm{DCN}$. First, the three $J=2 \leftarrow 1 R$-branch transitions were measured by Thomas et al. (1955) for both $\mathrm{CH}_{2} \mathrm{DCN}$ and $\mathrm{CHD}_{2} \mathrm{CN}$, so that only the rotational constants $B$ and $C$ were deduced approximately. Halonen \& Mills (1978) recorded about fifty microwave lines of $\mathrm{CHD}_{2} \mathrm{CN}$ between 18 and $40 \mathrm{GHz}$. They extended the determination of the spectroscopic parameters to all three rotational constants, as well as five quartic and four sextic distortion constants. However, based on the same measurements, an alternative fit has recently been proposed by H. S. P. Müller in the CDMS database using only two sextic distortion constants. No other rotational spectra of $\mathrm{CHD}_{2} \mathrm{CN}$ in a higher spectral region have been reported up to now. As for $\mathrm{CH}_{2} \mathrm{DCN}$, the spectroscopic data from laboratory measurements are more extended. Its ground-state rotational spectrum, as well as those of ${ }^{13} \mathrm{C}$ and ${ }^{15} \mathrm{~N}$ isotopologues, was measured by Le Guennec et al. (1992) from 120 to $470 \mathrm{GHz}$. Recently, Müller et al. (2009) have extended the data set up to about 1.2 THz. However, since $\mathrm{CH}_{2} \mathrm{DCN}$ was studied in natural abundance, they were not able to observe transitions with high quantum number $K_{a}$. The aim of this work is to extend the frequency range of measurements of $\mathrm{CHD}_{2} \mathrm{CN}$ to provide accurate predictions for astrophysical research and to complete the measurements of $\mathrm{CH}_{2} \mathrm{DCN}$.

With regard to astrophysical observations, $\mathrm{CH}_{2} \mathrm{DCN}$ was detected toward the hot core Orion-IRc2 and tentatively towards G34.3 by Gerin et al. (1992), which constitutes the unique published detection. They reported a $\mathrm{D} / \mathrm{H}$ ratio of about 0.01 that is subject to uncertainties, such as line opacity and beam dilution (uncertainty in the emission region), referred to in the text. For example, the authors give a minimum ratio of 0.004 if the emission of all isotopes comes only from the central 1 " region. Recent interferometric observations of Orion BN/KL by Peng et al. (2012) give a lower deuteration ratio for methanol $\left(\mathrm{CH}_{2} \mathrm{DOH} / \mathrm{CH}_{3} \mathrm{OH}\right)$ abundance of around $10^{-3}$. Further studies of $\mathrm{CH}_{2} \mathrm{DCN}$ would, therefore, be interesting for clearing up the uncertainties in its abundance, and these should involve ideally states of widely differing energy. No identification of $\mathrm{CHD}_{2} \mathrm{CN}$ has been reported to date, which reflects the lower abundance but also the lack of a complete spectral database to help in the identification. The possibility of detecting doubly deuterated methyl cyanide is especially interesting by interferometric observations of the hot corinos of low-mass protostars. In low-mass protostars, deuteration ratios are reported to be significantly higher than in high-mass ones, as shown for example in Parise et al. (2006).

Explanations of this high deuteration in terms of current gas and grain models show multiple inconsistencies (see for example Ratajczak et al. 2011). The deuteration, for at least two species (formaldehyde and methanol) is shown to depend critically on the precollapse phase and on D and $\mathrm{H}$ abstraction and substitution reactions (Taquet et al. 2012). Specifically, in the latter reference it is shown that the different deuterated species are formed at different times, so that their measure helps reconstruct the past history of the protostar. In the case of methyl cyanide, measuring the singly and doubly deuterated abundances would also help in understanding the relative contribution of the gas versus grain surface synthesis of this species (see Bottinelli et al. 2008 for a discussion of the various specific possibilities mentioned in the literature). It is, therefore, important to obtain accurate frequencies for both $\mathrm{CH}_{2} \mathrm{DCN}$ and $\mathrm{CHD}_{2} \mathrm{CN}$ for as many transitions as possible, to confirm or exclude their presence in radio-astronomical spectra (in particular of ALMA, in spectral surveys and/or for modeling confusion-limited detections) and to calculate significant maximum abundance limits in the case of non detection.

\section{Experimental}

The submillimeter-wave measurements (150-945 GHz) were performed using the Lille spectrometer (Motiyenko et al. 2010) based only on solid-state sources. The frequency of an Agilent synthesizer E8257D (12.5-17.5 GHz) was first multiplied by six and amplified by a Spacek active sextupler providing output power of $+15 \mathrm{dBm}$ in the $W$-band range $(75-110 \mathrm{GHz})$. This power is high enough to use passive Schottky multipliers $(\times 2$, $\times 3, \times 5, \times 3 \times 2, \times 3 \times 3$ ) from Virginia Diodes Inc in the next stage of the frequency multiplication chain. As a detector we used an InSb liquid He-cooled bolometer from QMC Instruments Ltd. To improve the sensitivity of the spectrometer, the sources were frequency-modulated at $10 \mathrm{kHz}$, and a $2 \mathrm{f}$ phase-sensitive detection was used. The absorption cell is a stainless-steel tube $(6 \mathrm{~cm}$ diameter, $220 \mathrm{~cm}$ long). The sample pressure during measurements was about 1.5 Pa (15 $\mu \mathrm{bar})$, and the linewidth was limited by Doppler broadening. Measurements were taken at room temperature. The accuracy of the measurements for isolated lines is estimated to be $30 \mathrm{kHz}$ up to $660 \mathrm{GHz}$ and $50 \mathrm{kHz}$ from 660 to $945 \mathrm{GHz}$. However, if the lines were blended or had a poor signal-to-noise ratio, they were given an uncertainty of 100 or even $200 \mathrm{kHz}$.

To synthesize the deuterated samples, the preparation of Anthoni and Nielsen (Anthoni \& Nielsen 1984) was modified (see Appendix A).

\section{Analysis and discussion}

Both $\mathrm{CH}_{2} \mathrm{DCN}$ and $\mathrm{CHD}_{2} \mathrm{CN}$ are near prolate asymmetric top molecules with $\kappa=-0.9973$ and -0.9965 , respectively. The parent molecule $\mathrm{CH}_{3} \mathrm{CN}$ or the totally deuterated $\mathrm{CD}_{3} \mathrm{CN}$ are symmetric tops, with the dipole moment along the symmetry (molecular) axis. The substitution of one $\mathrm{H}$ atom by $\mathrm{D}$ in the case of $\mathrm{CH}_{2} \mathrm{DCN}$, or one $\mathrm{D}$ by $\mathrm{H}$ in the other case, gives rise to a small rotation of the dipole moment from the molecular axis. This shift leads to $\mathrm{CH}_{2} \mathrm{DCN}$ having a much smaller $\mu_{b} \approx 0.12 \mathrm{D}$ component, as well as a large dipole moment component $\mu_{a}(\approx 3.92 \mathrm{D})$ along the molecular axis. Similarly, $\mu_{c} \approx 0.11 \mathrm{D}$ for $\mathrm{CHD}_{2} \mathrm{CN}$. In the ground vibrational state, these two species therefore exhibit strong $a$-type transitions and very weak $b$ (or respectively $c$ )-type transitions. The dipole moments are taken from a recent entry in the CDMS database and were derived from the value determined for the main isotopologue by Gadhi et al. (1984) taking the rotation of the inertial axes into account. The selection rule for the quantum number $J(\Delta J=0, \pm 1$ corresponding to $Q, R$ and $P$ branches, respectively) is completed by the selection rule for $K_{a}$ and $K_{c}$ that depends on the non zero components of the dipole moment (i.e. $\mu_{a} \neq 0: \Delta K_{a}=0, \pm 2 \ldots$; 
L. Nguyen et al.: Extension of the millimeter- and submillimeter-wave spectral databases of deuterated methyl cyanides

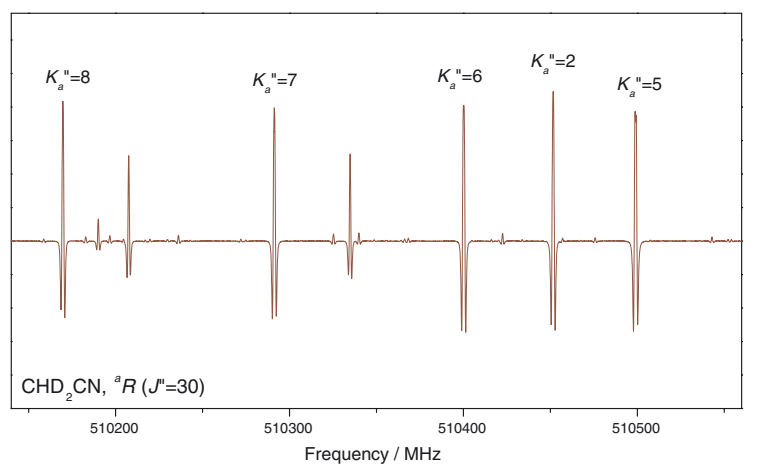

Fig. 1. Part of the submillimeter spectrum of $\mathrm{CHD}_{2} \mathrm{CN}$.

$\Delta K_{c}= \pm 1, \pm 3 \ldots ; \mu_{b} \neq 0: \Delta K_{a}= \pm 1, \pm 3 \ldots ; \Delta K_{c}= \pm 1, \pm 3 \ldots$ $\mu_{c} \neq 0: \Delta K_{a}= \pm 1, \pm 3 \ldots ; \Delta K_{c}=0, \pm 2 \ldots$ (Kroto 1975)). The majority of our measured lines were $a$-type transitions belonging to the $R$-branch with $\Delta K_{a}=0$ and $\Delta K_{c}=1$ that are the strongest in the frequency range measured. The $a$-type transitions with $\Delta K_{a}= \pm 2$ are very weak for asymmetric molecules close to the prolate symmetric limit, so were not measured. Some very weak $b$-type (or $c$-type) transitions could be observed but their identification demanded more caution.

Figure 1 gives an example of spectra recorded around $510 \mathrm{GHz}$ for $\mathrm{CHD}_{2} \mathrm{CN}$. The five labeled lines are $a$-type $R$-branch transitions with $J^{\prime \prime}=30$ and different $K_{a}^{\prime \prime}$ as given in the figure. The remaining unlabeled transitions (that probably correspond to the excited vibrational states) have not been assigned in the present work.

With the ASFIT program ${ }^{1}$, the identified transitions were fitted to the asymmetric top S-reduction of the rotational Hamiltonian in the $I^{r}$ representation (Watson 1977). The asymmetric top A-reduction was also tried but did not give a better fit. Predictions were made using the SPCAT program (Pickett et al. 1998) with the parameter set resulting from ASFIT and the dipole moments reported above.

\section{1. $\mathrm{CHD}_{2} \mathrm{CN}$}

Our initial identifications were based on the predictions that we made using the measurements of Halonen \& Mills (1978) between 18 and $40 \mathrm{GHz}$. First, we identified the strong $a$-type transitions of the $R$-branch from 150 to $250 \mathrm{GHz} .32$ lines (with $J^{\prime \prime}$ varying from 9 to 14 and $K_{a}^{\prime \prime}$ from 0 to 6) were measured with a systematic shift from the predictions (for example, about $3 \mathrm{MHz}$ at $J^{\prime \prime}=14$ and $K_{a}^{\prime \prime}=6$ ). These newly identified lines were then included in the fit. They fitted correctly, and the shifts decreased to below the experimental uncertainty. Next, we identified lines by progressively extending the frequency and systematically increasing $K_{a}$. At $K_{a}^{\prime \prime}=17$, we observed a significant disagreement between the measured and predicted frequencies of the transitions with $J^{\prime \prime}=29-37$ and $J^{\prime \prime}=49-54$. The maximum differences were observed for two transitions $J=37 \leftarrow 36$ (about $3 \mathrm{MHz}$ ) and $J=38 \leftarrow 37$ (about $7 \mathrm{MHz}$ ), while the others (of from 0.1 to $0.9 \mathrm{MHz}$ ) were also significant compared to the estimated experimental uncertainty of $0.05 \mathrm{MHz}$. This discrepancy between measurement and prediction was not resolved by adding more lines to refine parameters. Reasonable agreement was obtained for 14 transitions with $J^{\prime \prime}=18-28$, as well as with $J^{\prime \prime}=47$ (other $J^{\prime \prime}$ values were not observed). In the

\footnotetext{
1 Kisiel, PROSPE,

http://www.ifpan.edu.pl/ kisiel/prospe.htm
}

final analysis, transitions with $K_{a}^{\prime \prime}=17$ and shifted by more than $0.1 \mathrm{MHz}$ were omitted from the fit, and assumed perturbed (see Sect. 3.3). Transitions with $K_{a}^{\prime \prime}=18$ to 20 fitted correctly. Transitions with $K_{a}^{\prime \prime}=21,22$, and 23 were also observed, but no possible combination of parameters was found that allowed to fit them correctly. Lines of higher $K_{a}$ were too weak to be observed, so the highest $K_{a}$ included in the final fit is 20 , with a maximum $J$ of 56 .

The $c$-type transitions belonging to the $R$ - and $Q$-branches were then identified and progressively included in the fit, but only a very minor improvement was seen in the determined spectroscopic parameters. On the other hand, including 11 very weak $c$-type $P$-branch transitions (with $\Delta K_{a}=1$ and $\Delta K_{c}=-2$ ) allowed us to determine the sextic centrifugal distortion constant $H_{K}$.

Table 1 gives the rotational and centrifugal distortion parameters of $\mathrm{CHD}_{2} \mathrm{CN}$ in comparison with two lists of previously available parameters; one resulting from the fit by Halonen \& Mills (1978) and the other from a recent alternative fit given on the CDMS database ${ }^{2}$ and based on the same experimental data. In our work six sextic centrifugal distortion constants (all except $h_{3}$ ) were determined reliably. As mentioned previously, $H_{K}$ could also be determined thanks to a small number of $c$-type transitions, and its value $(49.9 \mathrm{~Hz})$ was very different from the $-1941 \mathrm{~Hz}$ obtained by Halonen \& Mills (1978) but very close to the $51 \mathrm{~Hz}$ of the parent molecule $\mathrm{CH}_{3} \mathrm{CN}$ estimated by Müller et al. (2009). This parameter was appropriately constrained to zero in the CDMS fit. We observed close agreement between our parameters and those that were determined in the CDMS fit, pointing out the very good choice made in the latter. For example, while two values of $H_{J K}$ and $h_{2}$ provided by the CDMS are close to those obtained in the present work, all four sextic $H_{K J}, H_{K}, h_{1}$, and $h_{2}$ distortion constants determined by Halonen \& Mills (1978) show significant disagreements. Similarly, agreements between the predictions in CDMS and our measurements are closer than those based on the original fit. For example, the CDMS prediction of the $a$-type transition with $J^{\prime \prime}=14$ and $K_{a}^{\prime \prime}=6$ mentioned above differs by only about $200 \mathrm{kHz}$. Our work thus confirms the high quality of the original low-frequency data of Halonen \& Mills (1978) and of the fit carried out on the CDMS that exploits this data the most reliably. Our fit differs from the latter on being based not only on much higher frequency data but also on many lines containing higher $J$ and much higher $K_{a}$ transitions. Along with the full set of sextic paramaters, three octic $\left(L_{J J K}, L_{J K}, L_{K K J}\right)$ and two decatic $\left(P_{K J}, P_{K K J}\right)$ centrifugal distortion constants were also needed to correctly fit all of our data.

For predictions of lines of higher $J$ and moderate $K_{a}$ that are still strong at temperatures of the 100-200 K found typically in hot cores and corinos, the new data differ by 1 to several $\mathrm{MHz}$ with the present CDMS fit and these differences increase at higher frequency. The differences become important in the case such as for lower abundance species in star-forming regions of spectral congestion and multi-species analysis where best candidates for detection depend not only on absolute intensity but also on minimum convolution with other nearby lines.

The final fit given in Table B.1 (Appendix B) contains 773 lines ( 723 new lines) and 1177 transitions with 404 unresolved doublets. Only the first 15 lines are listed, but a full list of measurements is available in electronic form at the CDS. The measured transitions not included in the fit are also shown in the full Table B.1 (noted by "e").

2 http://www.astro.uni-koeln.de/cdms 
Table 1. $\mathrm{CHD}_{2} \mathrm{CN}$ rotational and centrifugal distortion parameters.

\begin{tabular}{|c|c|c|c|}
\hline & This work & Halonen \& Mills (1978) & CDMS database* \\
\hline$A / \mathrm{MHz}$ & $96500.2696(22)$ & 96500.2674 (373) & $96500.2310(530)$ \\
\hline$B / \mathrm{MHz}$ & 8320.07278 (10) & $8320.0735(30)$ & 8320.0706 (44) \\
\hline $\mathrm{C} / \mathrm{MHz}$ & $8164.42785(10)$ & $8164.4282(30)$ & 8164.4253 (44) \\
\hline$D_{J} / \mathrm{kHz}$ & $3.103685(65)$ & $3.0903(34)$ & $3.10466(259)$ \\
\hline$D_{J K} / \mathrm{kHz}$ & $126.7475(21)$ & $126.2140(808)$ & $126.833(47)$ \\
\hline$D_{K} / \mathrm{kHz}$ & $1206.37(24)$ & $1213.57(319)$ & 1202.06 (159) \\
\hline$d_{1} / \mathrm{kHz}$ & $-0.047756(23)$ & $-0.04786(14)$ & $-0.048006(47)$ \\
\hline$d_{2} / \mathrm{kHz}$ & $-0.0105627(84)$ & $-0.010712(15)$ & $-0.0105365(46)$ \\
\hline$H_{J} / \mathrm{Hz}$ & $0.000472(11)$ & 0.0 & 0.0 \\
\hline$H_{J K} / \mathrm{Hz}$ & $0.72260(83)$ & 0.0 & $0.7172(188)$ \\
\hline$H_{K J} / \mathrm{Hz}$ & $0.774(21)$ & $60.14(418)$ & 0.0 \\
\hline$H_{K} / \mathrm{Hz}$ & $49.9(67)$ & $-1941(275)$ & 0.0 \\
\hline$h_{1} / \mathrm{mHz}$ & $-0.0615(65)$ & $-0.40(3)$ & 0.0 \\
\hline$h_{2} / \mathrm{mHz}$ & $0.0643(43)$ & $0.26(1)$ & $0.0637(64)$ \\
\hline$L_{J J K} / \mathrm{mHz}$ & $-0.00472(11)$ & & \\
\hline$L_{J K} / \mathrm{mHz}$ & $-0.0451(43)$ & & \\
\hline$L_{K K J} / \mathrm{mHz}$ & $-0.590(86)$ & & \\
\hline$P_{K J} / \mu \mathrm{Hz}$ & $0.2188(83)$ & & \\
\hline$P_{K K J} / \mu \mathrm{Hz}$ & $-8.27(11)$ & & \\
\hline Freq $_{\max }{ }^{a} / \mathrm{MHz}$ & 940798.15 & 39473.20 & 39473.20 \\
\hline nbr of transitions ${ }^{b}$ & 1177 & 50 & 50 \\
\hline$J_{\max }^{\prime \prime}, K_{a \max }^{\prime \prime}$ & 56,20 & 53,4 & 53,4 \\
\hline $\mathrm{rms}^{d} / \mathrm{kHz}$ & 30.6 & 26.2 & 21.5 \\
\hline $\mathrm{wrms}^{e}$ & 0.864 & & 0.649 \\
\hline
\end{tabular}

Notes. ${ }^{(*)}$ Revised fit by H. S. P. Müller using the data of Halonen \& Mills (1978), ${ }^{(a)}$ maximum line frequencies assigned; ${ }^{(b)}$ number of transitions included in the fit; ${ }^{(c)}$ maximum values of $J$ and $K_{a}$ (of lower state); ${ }^{(d)}$ standard deviation of fit; ${ }^{(e)}$ weighted standard deviation of fit.

\section{2. $\mathrm{CH}_{2} \mathrm{DCN}$}

Since an extensive set of measurements up to $1.2 \mathrm{THz}$ was already available by Müller et al. (2009), identification of $a$-type transitions was immediate. Besides the measurements of the authors (10 lines recorded in Cologne and 109 lines at JPL), the previous data set contained data from Thomas et al. (1955) and from Le Guennec et al. (1992). We decided to measure all possible transitions, so we remeasured lines already cited in the previous work that were within our frequency scans. As expected, the difference between the two sets of measurements was within the experimental uncertainty. The previous data were included in the fit without changes except for the remeasured lines that were replaced by the new ones. We then proceeded to measure lines with $K_{a}$ greater than ten, most of which had not been observed previously because of low sensitivity. Measurements were taken up to $K_{a}^{\prime \prime}=20$ and $J^{\prime \prime}=53$.

The problems with fitting observed for $\mathrm{CHD}_{2} \mathrm{CN}$ occurred for $\mathrm{CH}_{2} \mathrm{DCN}$ at lower $K_{a}$. We were not able to simultaneously fit transitions with $K_{a}^{\prime \prime}=14$ and $K_{a}^{\prime \prime}=15$ having high $J$ $\left(J^{\prime \prime}=45-51\right)$. A perturbation was again assumed, leading to the shift of all or some of these transitions. Since it was not possible to determine which of the transitions were unaffected, we decided to omit them all from the final fit. We were then, however, able to fit all the measured transitions with $K_{a}^{\prime \prime}=16$ correctly. We were also able to observe transitions with $K_{a}^{\prime \prime}=17-20$; however, no possible combination of parameters allowed us to fit these transitions correctly. This appeared similar to the problems encountered for fitting transitions with $K_{a}^{\prime \prime}>20$ for $\mathrm{CHD}_{2} \mathrm{CN}$ as described above.

To determine the $K$-dependent parameters that could not be determined from the $a$-type $R$-branch $\Delta K_{a}=0$ transitions, we also searched for $b$-type transitions. Only transitions belonging to the $R$-branch with $K_{a}=1 \leftrightarrow 0$ (13 transitions) and $K_{a}=$ $2 \leftarrow 1$ (4 transitions) were strong enough to be identified and measured. The resulting rotational, centrifugal distortion constants are given in Table 2. This is also the set that allows the best comparison with the di-deuterated and main isotopologues, as well as with previous results. Other parameter sets were tried with similar or worse results. A further discussion on the parameters is given in Sect. 3.3. The $D_{K}$ parameter was determined, and its value $(2.22 \mathrm{MHz})$ is bigger than the $1.97 \mathrm{MHz}$ fixed by Müller et al. (2009) and also than the $1.83 \mathrm{MHz}$ obtained by Le Guennec et al. (1992). The higher order $K$-dependent parameters, such as $H_{K}$ and $L_{K}$ were not determined reliably by the limited number of $b$-type transitions we identified. We therefore fixed $H_{K}=100 \mathrm{~Hz}$ to be consistent with Müller et al. (2009), and a comparison of the derived parameters is given in Table 2. Additionally, decatic centrifugal distortion constants $P_{K J}$ and $P_{K K J}$ were needed to correctly fit our data that include high $K_{a}$ transitions. In the analysis of Müller et al. (2009), two very weak $K_{a}=1 \leftrightarrow 2 b$-type transitions measured by Le Guennec et al. (1992) were excluded: one (the $9_{2,8} \leftarrow 10_{1,9}$ ) that did not fit and one $\left(31_{1,31} \leftarrow 30_{2,28}\right)$ that the authors did not trust. After including our $b$-type transitions, neither of these latter lines fitted correctly.

The final fit given in Table B.2 (Appendix B) contains 491 lines (307 new lines) and 733 transitions with 242 unresolved doublets. Only the first 14 lines are listed, but a full list of measurements is available in electronic form at the CDS.

\subsection{Discussion}

The weighted standard deviation of the fits is 0.864 for $\mathrm{CHD}_{2} \mathrm{CN}$ and 0.807 for $\mathrm{CH}_{2} \mathrm{DCN}$, indicating that, on average, the data have been fitted within experimental uncertainties, and that the latter may be slightly overestimated.

The analysis section reports a failure in fitting for some transitions above $K_{a}^{\prime \prime}=17$ for $\mathrm{CHD}_{2} \mathrm{CN}$ and $K_{a}^{\prime \prime}=14$ for $\mathrm{CH}_{2}$ DCN. An analogous shift from the predictions has already 
Table 2. $\mathrm{CH}_{2} \mathrm{DCN}$ rotational and centrifugal distortion parameters.

\begin{tabular}{lcc}
\hline \hline & This work & Müller et al. (2009) \\
\hline$A / \mathrm{MHz}$ & $121074.919(10)$ & $121074.677(20)$ \\
$B / \mathrm{MHz}$ & $8759.19798(12)$ & $8759.19794(23)$ \\
$C / \mathrm{MHz}$ & $8608.54244(11)$ & $8608.54255(24)$ \\
$D_{J} / \mathrm{kHz}$ & $3.469947(55)$ & $3.469982(137)$ \\
$D_{J K} / \mathrm{kHz}$ & $143.3383(60)$ & $143.3361(138)$ \\
$D_{K} / \mathrm{kHz}$ & $2224.2(21)$ & 1970.0 \\
$d_{1} / \mathrm{kHz}$ & $-0.079204(23)$ & $-0.079164(46)$ \\
$d_{2} / \mathrm{kHz}$ & $-0.004876(16)$ & $-0.004776(35)$ \\
$H_{J} / \mathrm{Hz}$ & $0.0004003(82)$ & $0.0004004(191)$ \\
$H_{J K} / \mathrm{Hz}$ & $0.8437(18)$ & $0.8375(49)$ \\
$H_{K J} / \mathrm{Hz}$ & $1.150(91)$ & $1.688(192)$ \\
$H_{K} / \mathrm{Hz}$ & 100. & 100. \\
$h_{1} / \mathrm{mHz}$ & $0.1544(53)$ & $0.1490(84)$ \\
$L_{J J K} / \mathrm{mHz}$ & $-0.00565(20)$ & $-0.00537(55)$ \\
$L_{J K} / \mathrm{mHz}$ & $0.017(13)$ & $0.058(28)$ \\
$L_{K K J} / \mathrm{mHz}$ & $-2.84(52)$ & $-10.82(68)$ \\
$P_{K J} / \mu \mathrm{Hz}$ & $0.324(39)$ & \\
$P_{K K J} / \mu \mathrm{Hz}$ & $-28.3(10)$ & \\
$\mathrm{Freq}_{\max }{ }^{\mathrm{M} H \mathrm{MHz}}$ & 1196285.09 & 1196285.09 \\
$\mathrm{nbr}$ of transitions ${ }^{b}$ & 733 & 274 \\
$J_{\max }^{\prime \prime}, K_{a \max }^{\prime \prime}$ & 68,16 & 68,14 \\
$\mathrm{rms}^{d} / \mathrm{kHz}$ & 57.7 & \\
${ }_{\mathrm{wrms}}{ }^{e}$ & 0.808 & 0.740 \\
\hline
\end{tabular}

Notes. ${ }^{(a)}$ Maximum line frequencies assigned; ${ }^{(b)}$ number of transitions included in the fit; ${ }^{(c)}$ maximum values of $J$ and $K_{a}$ (of lower state); ${ }^{(d)}$ standard deviation of fit; ${ }^{(e)}$ weighted standard deviation of fit.

been shown in the work of Simečková et al. (2004) for two transitions $J=39 \leftarrow 38, K=14$ and $J=40 \leftarrow 39, K=14$ of $\mathrm{CH}_{3} \mathrm{CN}$ in the ground vibrational state. The authors successfully explained this shift by resonances between the ground state rotational levels with $J>34, K=14$ and the $+l$ levels of the $v_{8}$ excited vibrational state with $K=12$. We assumed that a similar phenomenon occurred here for the deuterated isotopologues and that the problems observed with the fit for high $K_{a}$ arise from an interaction between high rotational energy levels in the vibrational ground state and other levels in an excited vibrational state. There are several low-energy excited vibrational states of $\mathrm{CH}_{3} \mathrm{CN}$, and the two lowest $v_{8}=1$ and $v_{8}=2$ are, respectively, at $365 \mathrm{~cm}^{-1}$ (Koivusaari et al. 1992) and $717 \mathrm{~cm}^{-1}$ (Tolonen et al. 1993) from the ground state well below, for example, the maximum rotational energy $2175 \mathrm{~cm}^{-1}$ of $\mathrm{CH}_{2} \mathrm{DCN}$ at $J^{\prime \prime}=48$ and $K_{a}^{\prime \prime}=20$ that we measured in the vibrational ground state. We were not able to find precise energies for similar vibrations in the deuterated isotopologues, so that a detailed analysis of perturbations was not possible. Furthermore, such an analysis is outside the main objectives of this publication since the lines in question are weak so not the best candidates for astrophysical use. Lines assumed to be perturbed have therefore been excluded from the fit but given in the data for future use.

Since both isotopologues are strongly prolate, with the $A$ rotational constant more than ten times larger than $B$, the energy of the rotational levels increases much more rapidly with $K_{a}$ than with $J$. This explains the lower limit in $K_{a}$ that could be measured compared to $J$, since the intensity decreases more rapidly with an increase in the former. It could also explain the limitation in $K_{a}$ for lines that could be included in the fit because of the increased possibility of resonance with a rovibrational level at higher energy. Similarly that the $A$ constant of $\mathrm{CH}_{2} \mathrm{DCN}$ is $25 \%$ larger than that of $\mathrm{CHD}_{2} \mathrm{CN}$ explains why $K_{a}$ is more limited for the former. However, lines of somewhat lower $K_{a}$ may show the same effect at high $J$. For example, the transition $J=50 \leftarrow 49$,
$K_{a}^{\prime \prime}=13$ is observed with the shift of $0.2 \mathrm{MHz}$, which is slightly higher than those obtained for the other $K_{a}^{\prime \prime}=13$ transitions.

Since perturbation depends on the (accidental) presence of a close-by lying rovibrational level, then there may be areas in energy where many lines are shifted and others where they are not. This is most clearly seen for $\mathrm{CHD}_{2} \mathrm{CN}$ where many lines cannot be fitted for $K_{a}^{\prime \prime}=17$, but fitting was then possible up to $K_{a}^{\prime \prime}=20$. Furthermore, even in the same energy region, some lines may be perturbed and others not. Above a certain value of $K_{a}^{\prime \prime}$ (22 for $\mathrm{CHD}_{2} \mathrm{CN}$ and 18 for $\mathrm{CH}_{2} \mathrm{DCN}$ ) it was not possible to fit the measured lines correctly. It is difficult to know whether to attribute this to perturbations, a breakdown in the approximations used in the calculations of energy levels, or too limited a number of measurements to correctly determine the higher order constants combined with an inability to correctly determine which lines are perturbed and which are not.

In principle, the more lines with high $K_{a}$ that are included in the fit, the better the determination of the higher order parameters and the more precise the prediction of other higher $K_{a}$ transitions. However, including lines with high $K_{a}$ demanded particular attention. We observed that lines with $K_{a}^{\prime \prime}=17$ for $\mathrm{CH}_{2} \mathrm{DCN}$ could be fit acceptably with enough parameters. However, we were not satisfied with the fit for several reasons. First, the prediction of higher $K_{a}$ lines is then degraded. Second, the higher-order centrifugal distortion constants change their value significantly or become undetermined when these transitions are included. Third, some lines of lower $K_{a}$ that fit previously become badly predicted. This last problem also affected the $K_{a}^{\prime \prime}=$ 21 transitions of $\mathrm{CHD}_{2} \mathrm{CN}$. For these reasons we preferred the fit for $\mathrm{CH}_{2} \mathrm{DCN}$ where lines are only included up to $K_{a}^{\prime \prime}=16$. Similarly, only lines up to $K_{a}^{\prime \prime}=20$ are fit for $\mathrm{CHD}_{2} \mathrm{CN}$.

It should be noted that two decatic centrifugal distortion constants $P_{K J}$ and $P_{K K J}$ were particularly needed by our high $K_{a}$ measured transitions (from $K_{a}^{\prime \prime}=19$ of $\mathrm{CHD}_{2} \mathrm{CN}$ and from $K_{a}^{\prime \prime}=16$ of $\left.\mathrm{CH}_{2} \mathrm{DCN}\right)$. Furthermore, the higher order centrifugal constants $\left(H_{K J}\right.$, and especially $L_{J K}$ and $\left.L_{K K J}\right)$ vary somewhat according to the exact choice of lines included in the fit and should be regarded as "fitting constants" rather than having high physical signification. However, a careful choice of lines to include and of the exact parameters to be fitted leads to the best prediction of higher $K_{a}$ lines as shown by comparison with the measurements. These considerations concern only the lines of high $K_{a}$. Regardless of the exact (sensible) choice, the stronger lower $K_{a}$ lines that are candidate for a first astrophysical detection are always predicted at the same frequency.

\section{Conclusion}

The rotational spectra of singly and doubly deuterated methyl cyanides, $\mathrm{CH}_{2} \mathrm{DCN}$ and $\mathrm{CHD}_{2} \mathrm{CN}$, in the ground vibrational state have been recorded up to $945 \mathrm{GHz}$. The existing data set of $\mathrm{CHD}_{2} \mathrm{CN}$ has been extended greatly and permits reliable predictions for a first astronomical identification of this molecule. For $\mathrm{CH}_{2} \mathrm{DCN}$, the prediction has been improved for high values of $K_{a}$. For both isotopologues, the extension of our data in high $K_{a}$ transitions permitted observing an apparent interaction for the first time between the rotational energy levels of the ground and excited vibrational states, as already suggested for the parent species. This is an interesting spectroscopic aspect that could be pursued in later work.

Acknowledgements. This work was supported by the Centre National d'Etudes Spatiales (CNES) and the Action sur Projets de l'INSU, "Physique et Chimie du Milieu Interstellaire". We thank the Agence Nationale de la Recherche for financial support (project ANR-08-BLAN-0225) and in particular for financing the postdoctoral position of Linh Nguyen. 


\section{Appendix A: Synthesis of the deuterated isotopologues}

In a Schlenk flask, equipped with a stirring bar, was introduced powdered potassium cyanide $(1.17 \mathrm{~g}, 17.7 \mathrm{mmol})$, dry triethylene glycol $(2 \mathrm{ml})$, and deuteromethyl iodide $(2.00 \mathrm{~g}$, $14.0 \mathrm{mmol}$ ). The mixture was cooled to $77 \mathrm{~K}$, the flask was evacuated, and the stopcock was closed. The solution was then stirred for $6 \mathrm{~h}$ at $40{ }^{\circ} \mathrm{C}$. After cooling at room temperature, the flask was connected to a vacuum line $(0.1 \mathrm{mbar})$ equipped with two U-tubes with stopcocks (Fig. A.1). The first one was immersed in a bath at $-30{ }^{\circ} \mathrm{C}$ to condense the high boiling impurities and the second one in a bath at $-90{ }^{\circ} \mathrm{C}$ to selectively trap deuteroacetonitrile in a vacuum. Yield: $89 \%$. NMR data. Synthesis of $\mathrm{CH}_{2} \mathrm{DCN}:{ }^{1} \mathrm{H} \mathrm{NMR}\left(\mathrm{CDCl}_{3}, 400 \mathrm{MHz}\right) \delta 1.96$ $\left(\mathrm{t},{ }^{2} J_{\mathrm{HD}}=2.4 \mathrm{~Hz}\right) \cdot{ }^{13} \mathrm{C} \mathrm{NMR}\left(\mathrm{CDCl}_{3}, 100 \mathrm{MHz}\right) \delta 1.71$ $\left({ }^{1} J_{\mathrm{CD}}=20.7 \mathrm{~Hz}(\mathrm{t}),{ }^{1} J_{\mathrm{CH}}=135.0 \mathrm{~Hz}(\mathrm{t}), \mathrm{CH}_{2} \mathrm{D}\right) ; 116.5(\mathrm{~s}$, $\mathrm{CN})$. Synthesis of $\mathrm{CHD}_{2} \mathrm{CN}:{ }^{1} \mathrm{H} \mathrm{NMR}\left(\mathrm{CDCl}_{3}, 400 \mathrm{MHz}\right)$

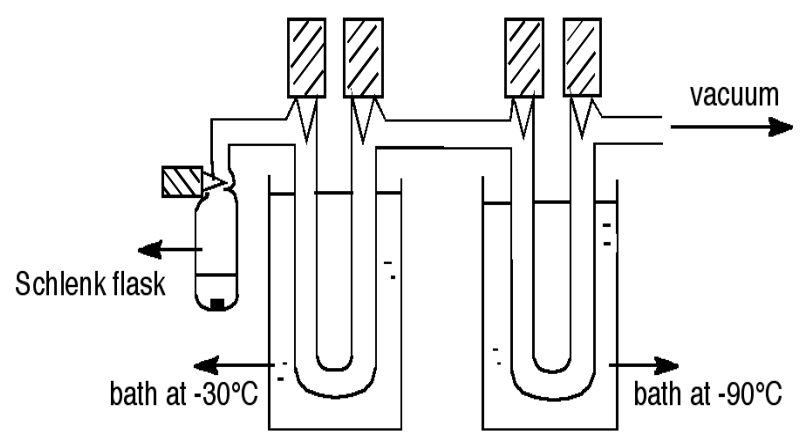

Fig. A.1. Synthesis scheme for the deuterated methyl cyanides.

$\delta 1.96\left(\mathrm{q},{ }^{2} J_{\mathrm{HD}}=2.4 \mathrm{~Hz}\right) .{ }^{13} \mathrm{C} \mathrm{NMR}\left(\mathrm{CDCl}_{3}, 100 \mathrm{MHz}\right) \delta 1.58$ $\left({ }^{1} J_{\mathrm{CD}}=21.0 \mathrm{~Hz}(\mathrm{q}),{ }^{1} J_{\mathrm{CH}}=135.0 \mathrm{~Hz}(\mathrm{~d}), \mathrm{CH}_{2} \mathrm{D}\right) ; 116.5$ $(\mathrm{s}, \mathrm{CN})$.

\section{Appendix B: Observed frequencies and fit of $\mathrm{CHD}_{2} \mathrm{CN}$ and $\mathrm{CH}_{2} \mathrm{DCN}$ in the ground state}

Table B.1. Observed frequencies and fit of $\mathrm{CHD}_{2} \mathrm{CN}$ in the ground state.

\begin{tabular}{ccccccccc}
\hline \hline$J^{\prime}$ & $K_{a}^{\prime}$ & $K_{c}^{\prime}$ & $J^{\prime \prime}$ & $K_{a}^{\prime \prime}$ & $K_{c}^{\prime \prime}$ & $\begin{array}{c}\text { Frequency } \\
(\mathrm{MHz})\end{array}$ & $\begin{array}{c}\text { Uncertainty } \\
(\mathrm{MHz})\end{array}$ & $\begin{array}{c}\text { Obs.-Calc. } \\
(\mathrm{MHz})\end{array}$ \\
\hline 10 & 1 & 10 & 9 & 1 & 9 & 164043.8545 & 0.0300 & -0.0041 \\
10 & 6 & 4 & 9 & 6 & 3 & $164742.8533+$ & 0.0300 & 0.0732 \\
10 & 6 & 5 & 9 & 6 & 4 & $164742.8533+$ & 0.0300 & 0.0732 \\
10 & 5 & 5 & 9 & 5 & 4 & $164771.0991+$ & 0.0300 & 0.0271 \\
10 & 5 & 6 & 9 & 5 & 5 & $164771.0991+$ & 0.0300 & 0.0271 \\
10 & 4 & 7 & 9 & 4 & 6 & $164794.7045+$ & 0.0300 & -0.0080 \\
10 & 4 & 6 & 9 & 4 & 5 & $164794.7045+$ & 0.0300 & -0.0080 \\
10 & 0 & 10 & 9 & 0 & 9 & 164798.6268 & 0.0300 & -0.0157 \\
10 & 3 & 8 & 9 & 3 & 7 & 164814.4499 & 0.0300 & 0.0812 \\
10 & 2 & 9 & 9 & 2 & 8 & 164817.3348 & 0.0300 & 0.0334 \\
10 & 2 & 8 & 9 & 2 & 7 & 164851.1481 & 0.0300 & -0.0190 \\
10 & 1 & 9 & 9 & 1 & 8 & 165599.7877 & 0.0300 & -0.0045 \\
37 & 5 & 32 & 38 & 4 & 34 & $166150.9717 \mathrm{c}$ & 0.0300 & 0.0654 \\
37 & 5 & 33 & 38 & 4 & 35 & $166164.1827 \mathrm{c}$ & 0.0300 & -0.0208 \\
17 & 0 & 17 & 16 & 1 & 15 & $180733.0036 \mathrm{c}$ & 0.0300 & 0.0456 \\
\hline
\end{tabular}

Notes. The full Table B.1 is available in electronic form at the CDS. A portion with only the 15 lowest frequency transitions is shown here for guidance regarding its form and content. Transitions are assigned with the rotational quantum numbers of the upper (first) and lower (second) levels. Observed frequencies are given. A "c" shows the $c$-type transitions. Transitions without "c" are of $a$-type. Sign "+" shows the blended transitions. An "e" (seen in the full table) shows the transitions observed but rejected from the fit as described in the text, because of the significant difference between observed and calculated frequencies; the observed minus calculated difference (Obs.-Calc.) is not given for these transitions.

Table B.2. Observed frequencies and fit of $\mathrm{CH}_{2} \mathrm{DCN}$ in the ground state.

\begin{tabular}{ccccccccc}
\hline \hline$J^{\prime}$ & $K_{a}^{\prime}$ & $K_{c}^{\prime}$ & $J^{\prime \prime}$ & $K_{a}^{\prime \prime}$ & $K_{c}^{\prime \prime}$ & $\begin{array}{c}\text { Frequency } \\
(\mathrm{MHz})\end{array}$ & $\begin{array}{c}\text { Uncertainty } \\
(\mathrm{MHz})\end{array}$ & $\begin{array}{c}\text { Obs.-Calc. } \\
(\mathrm{MHz})\end{array}$ \\
\hline 28 & 20 & 8 & 27 & 20 & 7 & $482765.4041 \mathrm{e}$ & 0.0500 & \\
28 & 20 & 9 & 27 & 20 & 8 & $482765.4041 \mathrm{e}$ & 0.0500 & \\
28 & 17 & 11 & 27 & 17 & 10 & $483686.7272 \mathrm{e}$ & 0.0300 & \\
28 & 17 & 12 & 27 & 17 & 11 & $483686.7272 \mathrm{e}$ & 0.0300 & -0.0416 \\
28 & 16 & 12 & 27 & 16 & 11 & $483953.9374+$ & 0.0300 & -0.0416 \\
28 & 16 & 13 & 27 & 16 & 12 & $483953.9374+$ & 0.0300 & 0.0576 \\
28 & 15 & 13 & 27 & 15 & 12 & $484203.4077+$ & 0.0300 & 0.0576 \\
28 & 15 & 14 & 27 & 15 & 13 & $484203.4077+$ & 0.0300 & -0.0195 \\
28 & 14 & 14 & 27 & 14 & 13 & $484435.8082+$ & 0.0300 & 0.0195 \\
28 & 14 & 15 & 27 & 14 & 14 & $484435.8082+$ & 0.0300 & 0.0300 \\
28 & 13 & 15 & 27 & 13 & 14 & $484651.8126+$ & 0.072 \\
28 & 13 & 16 & 27 & 13 & 15 & $484651.8126+$ & 0.0300 & 0.0072 \\
28 & 12 & 16 & 27 & 12 & 15 & $484851.5592+$ & 0.0300 & -0.0161 \\
28 & 12 & 17 & 27 & 12 & 16 & $484851.5592+$ & 0.0300 & -0.0161 \\
\hline
\end{tabular}

Notes. The full Table B.2 is available in electronic form at the CDS. A portion with only the 14 lowest frequency transitions is shown here for guidance regarding its form and content. Transitions are assigned with the rotational quantum numbers of the upper (first) and lower (second) levels. Observed frequencies are given. A "b" (seen in the full table) shows the $b$-type transitions. Transitions without "b" are of $a$-type. Sign "+" shows the blended transitions. An "e" shows the transitions observed but rejected from the fit as described in the text, because of the significant difference between observed and calculated frequencies; the observed minus calculated difference (Obs.-Calc.) is not given for these transitions. 
L. Nguyen et al.: Extension of the millimeter- and submillimeter-wave spectral databases of deuterated methyl cyanides

\section{References}

Anthoni, U., \& Nielsen, H. 1984, J. Labelled Cmpds. Radiopharm., 21, 375 Bottinelli, S., Ceccarelli, C., Neri, R., \& Williams, J. P. 2008, in Organic Matter in Space, Proc. IAU Symp., 251

Boucher, D., Burie, J., Bauer, A., Dubrulle, A., \& Demaison, J. 1980, J. Phys Chem. Ref. Data, 9, 659

Brown, F. X., Cosleou, J., Dangoisse, D., Demaison, J., \& Wlodarczak, G. 1989, J. Mol. Spectrosc., 134, 234

Cazzoli, G., \& Puzzarini, C. 2006, J. Mol. Spectrosc., 240, 153

Ceccarelli, C., Caselli, P., Herbst, E., Tielens, A. G. G. M., \& Caux, E. 2007, in Protostars and Planets V, eds. B. Reipurth, D. Jewitt, \& K. Keil (Tucson, AZ: Univ. Arizona Press), 951, 47

Chen, W., Bocquet, R., Boucher, D., Demaison, J., \& Wlodarczak, G. 1991, J. Mol. Spectrosc., 150, 470

Cosleou, J., Wlodarczak, G., J., B., \& Demaison, J. 1989, J. Mol. Spectrosc., 137,47

Cosleou, J., Lopéz, J. C., L, A. J., Wlodarczak, G., \& Demaison, J. 1991, J. Mol. Spectrosc., 149, 242

Demaison, J., Dubrulle, A., Boucher, D., \& Burie, J. 1979, J. Mol. Spectrosc., 76,1

Gadhi, J., Lahrouni, A., Legrand, J., \& Demaison, J. 1984, J. Chim. Phys., 92, 1984

Gerin, M., Combes, F., Wlodarczak, G., et al. 1992, A\&A, 259, L35

Halonen, L., \& Mills, I. M. 1978, J. Mol. Spectrosc., 73, 494
Koivusaari, M., Horneman, V. M., \& Antilla, R. 1992, J. Mol. Spectrosc., 152, 377

Kroto, H. W. 1975, in Molecular Rotation Spectra (John Wiley \& Sons Ltd)

Le Guennec, M., Wlodarczak, G., Burie, J., \& Demaison, J. 1992, J. Mol Spectrosc., 154, 305

Matsumura, C., Hirota, E., Oka, T., \& Morino, Y. 1962, J. Mol. Spectrosc., 9, 366

Müller, H. S. P., Drouin, B. J., \& Pearson, J. C. 2009, A\&A, 506, 1487

Motiyenko, R. A., Margulès, L., Alekseev, E. A., Guillemin, J. C., \& \& Demaison, J. 2010, J. Mol. Spectrosc., 264, 94

Parise, B., Ceccarelli, C., Tielens, A. G. G. M., et al. 2006, A\&A, 453, 949

Pearson, J. C., \& Müller, H. S. P. 1996, ApJ, 471, 1067

Peng, T. C., Despois, D., Brouillet, N., Parise, B., \& Baudry, A. 2012, A\&A, 543, A 152

Pickett, H. M., Poynter, R. L., Cohen, E. A., et al. 1998, J. Quant. Spec. Radiat. Transf., 60, 883

Ratajczak, A., Taquet, V., Kahane, C., et al. 2011, A\&A, 528, L13

Simečková, M., Urban, S., Fuchs, U., et al. 2004, J. Mol. Spectrosc., 226, 123

Tam, H., An, I., \& Roberts, J. A. 1988, J. Mol. Spectrosc., 129, 202

Taquet, V., Ceccarelli, C., \& Kahane, C. 2012, ApJ, 748, L3

Thomas, L. F., Sherrard, E. J., \& Sheridan, J. 1955, Trans. Faraday Soc., 51, 619

Tolonen, A., Koivusaari, M., Paso, R., et al. 1993, J. Mol. Spectrosc., 160, 554

Watson, J. K. G. 1977, in Vibrational Spectra and Structure (Amsterdam: Elsevier) 